\section{Effects of Fertilizer Type on Quality and Nutrient Content of Established Landscape Plants in Florida}

\author{
T.K. Broschat ${ }^{1,4}$, D.R. Sandrock ${ }^{2,3}$, M.L. Elliott ${ }^{1}$, \\ and E.F. Gilman ${ }^{2}$
}

ADDITIONAL INDEX wORDs. palms, turfgrass, woody ornamentals, Dypsis lutescens, Stenotaphrum secondatum, Nandina domestica, Canna $\times$ generalis, Pentas lanceolata, Allamanda cathartica, controlled release fertilizer, nitrogen, potassium, magnesium, iron, nutrient deficiencies

SumMARY. In a series of three experiments, st. augustinegrass (Stenotaphrum secundatum 'Floratam'), areca palm (Dypsis lutescens), canna (Canna $\times$ generalis 'Richard Wallace'), pentas (Pentas lanceolata), allamanda (Allamanda catbartica 'Hendersoni'), and nandina (Nandina domestica) were grown on highly leached sand soils in two locations in Florida. They were fertilized with typical turfgrass fertilizers having high nitrogen $(\mathrm{N})$-to-potassium $(\mathrm{K})$ ratios and no magnesium $(\mathrm{Mg})$, or several types of landscape palm fertilizers having low $\mathrm{N}: \mathrm{K}$ ratios and $100 \%$ of their $\mathrm{N}, \mathrm{K}$, and $\mathrm{Mg}$ in controlled release form. St. augustinegrass, pentas, nandina, and allamanda visual quality were similar for all fertilizer types tested. However, cannas and areca palms had higher visual qualities when fertilized with an $8 \mathrm{~N}-0.9 \mathrm{P}-10.0 \mathrm{~K}-4 \mathrm{Mg}$ palm fertilizer than with higher $\mathrm{N}: \mathrm{K}$ ratio turf fertilizers. High $\mathrm{N}: \mathrm{K}$ turf fertilizers resulted in $\mathrm{K}$ deficiency severity equivalent to that of unfertilized controls and $\mathrm{Mg}$ deficiency that was more severe than unfertilized areca palms.

$\mathrm{F}$ ertilization of established ornamental plants within the landscape has been given little attention by researchers to date. This may be because of a number of factors. Soils and climatic characteristics vary considerably among regions, and optimum fertilization recommendations will vary accordingly. A wide array of plant materials are grown in landscapes across the United States, and each type may have its own unique fertility requirements. Finally, some plant types, notably woody dicots, seem to grow satisfactorily in most soils without supplemental fertilization (Gilman, 1991).

One group of plants widely grown in tropical, subtropical,

This project was funded in part by the Florida Agricultural Experiment Station.

The authors wish to thank Anita Durden, William Latham, Susan Thor, Chris Harchick, and Patti Anderson for their assistance in these experiments.

${ }^{1}$ Fort Lauderdale Research \& Education Center, University of Florida, 3205 College Avenue, Fort Lauderdale, FL 33314

${ }^{2}$ Environmental Horticulture Department, University of Florida, Gainesville, FL 32611

${ }^{3}$ Current address: Department of Horticulture, Oregon State University, Corvallis, OR 97331.

${ }^{4}$ Corresponding author. E-mail: tkbr@ufl.edu.
Mediterranean, and warm temperate climates that is highly susceptible to unsightly, debilitating, and sometimes fatal nutritional deficiencies are palms (Elliott et al., 2004). Because landscape ornamental plants are grown for their appearance, the presence of nutritional deficiency symptoms can reduce their aesthetic value, in addition to affecting plant health. Although nutritional deficiencies in most plants merely reduce growth rate, yield, or visual quality, deficiencies of $\mathrm{K}$, manganese $(\mathrm{Mn})$, and boron (B) can be fatal in palms (Elliott et al., 2004), which are monocots possessing a single shoot meristem (Tomlinson, 1990). For the majority of ornamental palm species on most soil types, $\mathrm{K}$ deficiency is the most important nutrient deficiency in terms of impact on palm physical appearance and health (Elliott et al., 2004). Furthermore, high soil $\mathrm{N}$ concentrations are believed to accentuate $\mathrm{K}$ deficiencies in palms, presumably because of a dilution effect (Broschat, 2005a).

On highly leached, low cation exchange capacity (CEC) sandy soils, such as those commonly found within the Atlantic coastal plain of the southeastern United States, palms and plants in the Zingiberales are commonly deficient in $\mathrm{K}, \mathrm{Mg}$, iron $(\mathrm{Fe})$, $\mathrm{B}, \mathrm{Mn}$, and $\mathrm{N}$ (Broschat, 1992, 2005b); dicot trees, shrubs, and herbaceous ornamental plants show deficiencies of $\mathrm{Mg}, \mathrm{Fe}, \mathrm{Mn}, \mathrm{N}$, and occasionally $\mathrm{B}, \mathrm{K}$, or zinc $(\mathrm{Zn})$ (Dickey, 1977); and turfgrasses show deficiencies primarily of $\mathrm{N}, \mathrm{K}$, and $\mathrm{Fe}$ (Duble, 1996; Trenholm and Unruh, $2005 b)$. Water-soluble sources of $\mathrm{N}$, $\mathrm{K}, \mathrm{Mg}$, and $\mathrm{B}$ can be rapidly leached out of the low CEC sandy soils, typical of large portions of Florida, with regular irrigation or normal rainfall. Thus, controlled release sources of these elements have been found to be almost essential for treating or preventing deficiencies of these elements in palms on these soils (Broschat, 2005a). For maintenance of landscape palms in Florida, fertilizers having an analysis of $8 \mathrm{~N}-0.9 \mathrm{P}-$ $10.0 \mathrm{~K}-4 \mathrm{Mg}$ plus $\mathrm{Fe}, \mathrm{Mn}$, copper $(\mathrm{Cu}), \mathrm{Zn}$, and $\mathrm{B}$ with $100 \%$ of the $\mathrm{N}, \mathrm{K}$, and $\mathrm{Mg}$ in these blends in controlled release form are recommended (Broschat, 2005a; Knox et al., 2002). This low $\mathrm{N}: \mathrm{K}$ ratio analysis (2:3) differs greatly from the high $\mathrm{N}$ :K ratio (2:1 or higher) products commonly applied to landscape turfgrass in Florida (Trenholm and Unruh, 2005a). Furthermore, most landscape turfgrass fertilizers do not contain $\mathrm{Mg}$, and their $\mathrm{K}$ sources are water soluble.

\begin{tabular}{llll}
\hline $\begin{array}{l}\text { Units } \\
\begin{array}{l}\text { To convert U.S. to SI, } \\
\text { multiply by }\end{array}\end{array}$ & U.S. unit & SI unit & $\begin{array}{l}\text { To convert SI to U.S., } \\
\text { multiply by }\end{array}$ \\
\hline 0.3048 & $\mathrm{ft}$ & $\mathrm{m}$ & 3.2808 \\
0.0929 & $\mathrm{ft}^{2}$ & $\mathrm{~m}^{2}$ & 10.7639 \\
0.0283 & $\mathrm{ft}^{3}$ & $\mathrm{~m}^{3}$ & 35.3147 \\
3.7854 & $\mathrm{gal}$ & $\mathrm{L}$ & 0.2642 \\
2.54 & inch $(\mathrm{es})$ & $\mathrm{cm}$ & 0.3937 \\
1 & $\mathrm{meq} / 100 \mathrm{~g}$ & $\mathrm{cmol} \cdot \mathrm{kg}^{-1}$ & 1 \\
33.9057 & $\mathrm{oz} / \mathrm{yard}$ & $\mathrm{g} \cdot \mathrm{m}^{-2}$ & 0.0295 \\
1 & $\mathrm{ppm}$ & $\mathrm{mg} \cdot \mathrm{kg}^{-1}$ & 1 \\
1 & $\mathrm{ppm}$ & $\mu \mathrm{g} \cdot \mathrm{mL}^{-1}$ & 1
\end{tabular}


Palms in landscapes receiving high $\mathrm{N}: \mathrm{K}$ ratio turfgrass fertilizers have been observed to die from $\mathrm{N}$ induced $\mathrm{K}$ deficiency (Broschat, 2005a). Because palms often represent the most expensive component in a landscape, and the correction of existing deficiencies can take 1 to 3 years or longer, prevention of these deficiencies by proper fertilization is important. The application of a single fertilizer that addresses the nutritional needs of palms, as well as those of turfgrass and other landscape plants, would be convenient for application and advantageous for all types of plants growing within the mosaic of plant types characteristic of landscapes within the southern Atlantic coastal plain. The purpose of this study was to determine the effects of several types of fertilizers on the quality of palms, st. augustinegrass, and other landscape plants after establishment.

\section{Materials and methods}

A series of three experiments was established to determine the effects of no fertilizer, high $\mathrm{N}: \mathrm{K}$ ratio (2:1 or higher) turfgrass fertilizer, low $\mathrm{N}: \mathrm{K}$ ratio $(2: 3)$ palm fertilizer, or intermediate $\mathrm{N}: \mathrm{K}$ ratio $(\mathrm{l}: \mathrm{l})$ palm fertilizers on st. augustinegrass, a palm or other monocot with similar nutritional requirements, and a woody dicot species. The exact same products were not used in each experiment as experiments were started at different times and some materials became unavailable (Table 1 ). Fertilizers were substituted that had similar $\mathrm{N}: \mathrm{K}$ ratios and similar compositions.

Expt. 1 was conducted on established 'Floratam' st. augustinegrass turf in Davie, Florida (lat. $26^{\circ} 5^{\prime} 3.51^{\prime \prime} \mathrm{N}$, long. $\left.80^{\circ} 14^{\prime} 19.59^{\prime \prime} \mathrm{W}\right)$. This turfgrass was established in 1981, but received no regular fertilization before this experiment. It was planted on a Margate fine sand soil (siliceous, hyperthermic Mollic Psammaquent). This soil has a CEC of $5.6 \mathrm{meq} / 100 \mathrm{~g}$ and a pH of 6.2 (U.S. Department of Agriculture, 1984). The turfgrass received $\approx 60$ inches of rainfall annually plus irrigation only when signs of wilt were visible. Irrigation water was drawn from a pond and had a $\mathrm{pH}$ of 7.0 and contained $0.5 \mu \mathrm{g} \cdot \mathrm{mL}^{-1}$ of $\mathrm{K}$ and $1.4 \mu \mathrm{g} \cdot \mathrm{mL}^{-1}$ of $\mathrm{Mg}$.

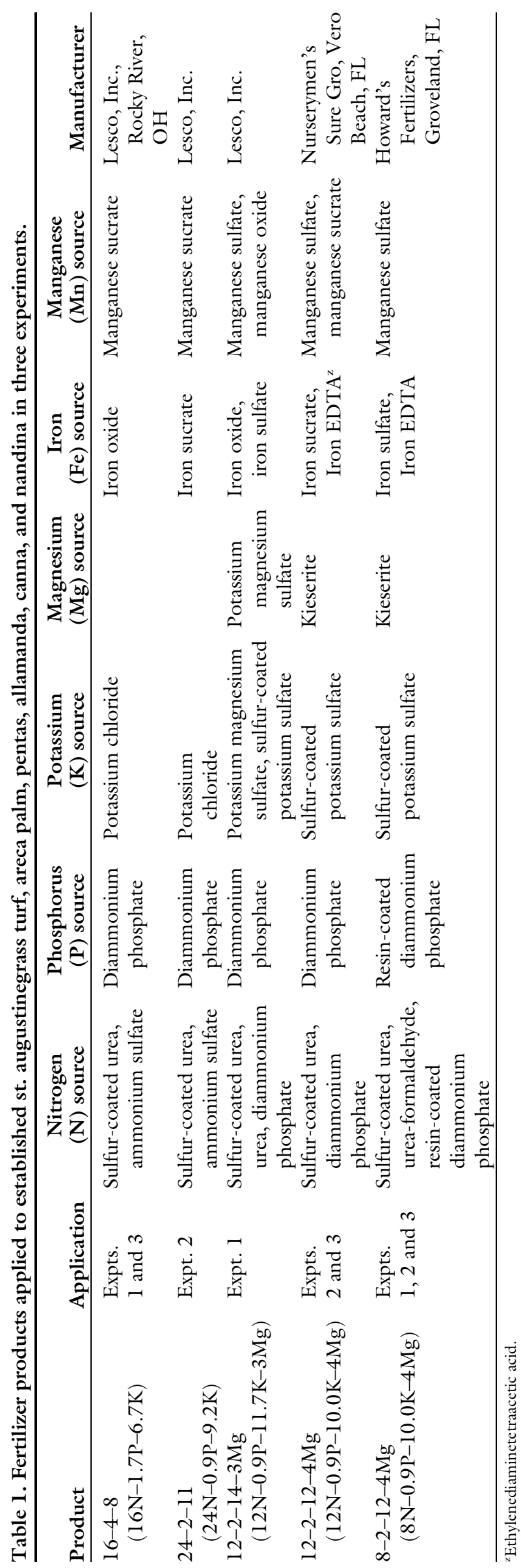


Four replicate plots, each $4 \mathrm{ft}$ wide by $30 \mathrm{ft}$ long and arranged in a randomized complete block design, were treated every 3 months beginning on 3 Nov. 2000 , with $4.9 \mathrm{~g} \cdot \mathrm{m}^{-2}$ $\mathrm{N}$ from: 1) a $16-4-8(16 \mathrm{~N}-1.7 \mathrm{P}-$ $6.7 \mathrm{~K})$ mostly water-soluble turf fertilizer plus $\mathrm{Mn}$ and $\mathrm{Fe}, 2$ ) an 8-2$12-4 \mathrm{Mg} \quad(8 \mathrm{~N}-0.9 \mathrm{P}-10.0 \mathrm{~K}-4 \mathrm{Mg})$ plus micronutrients controlled-release palm fertilizer, or 3) a 12-2-14$3 \mathrm{Mg}(12 \mathrm{~N}-0.9 \mathrm{P}-11.7 \mathrm{~K}-3 \mathrm{Mg})$ plus micronutrients partially controlledrelease palm fertilizer (Table 1 ). Taking into consideration the differences in the amounts of each fertilizer that were applied to achieve equal $\mathrm{N}$ amounts, the relative amounts of potassium oxide $\left(\mathrm{K}_{2} \mathrm{O}\right)$ applied in this experiment were $1 \times, 3 \times$, and $2.3 \times$ for treatments 1 through 3 , respectively. Control plots received no fertilizer. Fertilizers were applied using a calibrated rotary spreader, and the plots had 2 -ft-wide untreated buffer strips between them. Turf clippings were not removed from the plots after mowings. Plots were visually rated for overall quality (includes color and density) every 2 to 3 months by the same two investigators on a scale of 0 to $10(0=$ dead, $10=$ highest possible quality for the species) with means from the two investigators' ratings being used as data. Samples for foliar nutrient analyses were collected from the youngest fully expanded leaves before mowing on 3 May 2002, the termination of Expt. 1 (Broschat and Elliott, 2004). Leaf samples were not washed before analysis. Dried leaf samples were digested using a sulfuric acid-hydrogen peroxide method (Hach et al., 1987), with $\mathrm{N}$ determination by ammonia electrode (Greenberg and Eaton, 1992), $\mathrm{P}$ by the ascorbic acid method (Kuo, 1996), and other elements by inductively coupled plasma spectroscopy (Soltanpour et al., 1996).

Expt. 2 was planted on 16 July 2004 at a nearby site in Davie, Florida (lat. $26^{\circ} 5^{\prime} 5.60^{\prime \prime} \mathrm{N}$, long. $80^{\circ} 14^{\prime} 34.60^{\prime \prime} \mathrm{W}$ ) on the Margate fine sand soil described for Expt. 1. Three species of plants, areca palm, pentas, and allamanda were planted into $1-\mathrm{m}^{2}$ plots, each containing a single plant and separated by 1 -ft-wide buffer strips. All plants had been growing in \#l (2.4-L) plastic containers using a 5 pine bark:4 sedge peat: 1 sand (by volume) substrate. Fertilizer treatments were: 1) no fertilizer, 2) a 24-2-1 l (24N-0.9P-9.2K) plus micronutrients mostly water-soluble turf fertilizer, 3) the $8-2-12-4 \mathrm{Mg}$ palm fertilizer used in Expt. 1, or 4) a $12-2-12-4 \mathrm{Mg}(12 \mathrm{~N}-0.9 \mathrm{P}-10.0 \mathrm{~K}-$ $4 \mathrm{Mg}$ ) plus micronutrients mostly controlled-release palm fertilizer (Table 1). Taking into consideration the differing amounts of each product applied to achieve equal $\mathrm{N}$ amounts, the relative amounts of $\mathrm{K}_{2} \mathrm{O}$ applied in this Expt. were $1 \times, 3 \times$, and $2 \times$, for treatments 2 through 4 , respectively. All fertilizers were broadcast by hand uniformly over the $1-\mathrm{m}^{2}$ plot areas at a rate equivalent to $4.9 \mathrm{~g} \cdot \mathrm{m}^{-2} \mathrm{~N}$ per application; applications were made quarterly. There were six blocks containing three replicate plots per species. Treatments were arranged in a randomized complete block design.

All plots received $\approx 2 \mathrm{~cm}$ of water two times per week from overhead irrigation heads (K-Rain Proplus model 11003; K-Rain, West Palm Beach, FL) mounted on 3-ft-high risers spaced $30 \mathrm{ft}$ apart along the north and south perimeters of the experimental plot, in addition to natural rainfall. Plots in Expt. 2 were irrigated from the same pond used in Expt. 1. Weeds were controlled in the plots by manual removal and directed sprays with glyphosate.

After 2 years, total height and two perpendicular widths of pentas and allamanda plants were measured. Total plant size calculated as height $x$ width $1 \times$ width 2 . Total height, measured to the top of the tallest extended leaf, was used as the sole measure of plant size for areca palms. All plants were rated subjectively to the nearest tenth of a unit for severity of $\mathrm{N}, \mathrm{K}$, and $\mathrm{Mg}$ deficiencies on a scale of 1 to $5(\mathrm{l}=$ severe deficiency, $5=$ no visible deficiency symptoms). Symptom severity was based on overall foliage color intensity for $\mathrm{N}$, and proportion of the canopy displaying $\mathrm{K}$ or $\mathrm{Mg}$ deficiency symptoms. Samples consisting of the youngest fully expanded leaves on each shoot (middle leaflets of these leaves for areca palms) were collected for nutrient analysis. Leaf samples were not washed before analysis. Nutrients were analyzed as described for Expt. 1.

Expt. 3 was planted on 18 May 2004 on a Bonneau fine sand soil (loamy, siliceous, subactive, thermic Arenic Paleudults) near Gainesville,
Florida (lat. $29^{\circ} 37^{\prime} 24.96^{\prime \prime} \mathrm{N}$, long. $\left.82^{\circ} 21^{\prime} 13.02^{\prime \prime} \mathrm{W}\right)$. This soil had a $\mathrm{pH}$ of 7.8 and a CEC of $1.8 \mathrm{meq} /$ 100 g. One-square-meter plots, each containing a single plant of canna or nandina, and separated by 2 -ft-wide buffer strips, were used as experimental units. A randomized complete block design with three blocks and four replicate plots per species and fertilizer treatment was used. Fertilizer treatments were: 1) no fertilizer, 2 ) the 16-4-8 turf fertilizer used in Expt. 1, 3) the 8-2-12-4Mg palm fertilizer used in Expts. 1 and 2, or 4) or the $12-2-12-4 \mathrm{Mg}$ palm fertilizer used in Expt. 2. Taking into consideration the differing amounts of each product applied to achieve equal $\mathrm{N}$ amounts, the relative amounts of $\mathrm{K}_{2} \mathrm{O}$ applied in this Expt. were $1 \times, 3 \times$, and $2 \times$ for treatments 2 through 4 , respectively. Fertilizers were applied quarterly at a rate equivalent to 4.9 $\mathrm{g} \cdot \mathrm{m}^{-2} \mathrm{~N}$ per application and were broadcast by hand within the $1 \mathrm{~m}^{2}$ plot area.

Plots were irrigated with $2 \mathrm{~cm}$ of water two times per week with rotary heads located $30 \mathrm{ft}$ apart on the north and south perimeter of the experimental plot, in addition to natural rainfall of about 53 inches per year. The irrigation water was obtained from a well and had a $\mathrm{pH}$ or 7.5 and an electrical conductivity (EC) of 0.61 . It contained $118 \mu \mathrm{g} \cdot \mathrm{mL}^{-1} \mathrm{Ca}$, $3.9 \mu \mathrm{g} \cdot \mathrm{mL}^{-1} \mathrm{Mg}, 3.3 \mu \mathrm{g} \cdot \mathrm{mL}^{-1} \mathrm{Fe}$, $12.4 \mu \mathrm{g} \cdot \mathrm{mL}^{-1} \mathrm{Na}, 22.0 \mu \mathrm{g} \cdot \mathrm{mL}^{-1} \mathrm{Cl}$, and $6.8 \mu \mathrm{g} \cdot \mathrm{mL}^{-1}$ carbonates. Weeds were removed manually or by directed sprays with glyphosate within plots, and interplot buffer areas were covered with 2 -ft-wide woven polypropylene ground cloth.

After 2 years, plots were measured for total plant height and two perpendicular widths, with plant size index calculated as in Expt. 2. Cannas were rated subjectively for severity of $\mathrm{N}, \mathrm{K}$, and $\mathrm{Mg}$ deficiencies, whereas nandinas were rated for $\mathrm{N}, \mathrm{Mg}$, and Fe deficiencies as in Expt. 2. Leaf samples were collected for nutrient analysis as in Expt. 2. However, because the irrigation water left visible $\mathrm{Fe}$ and $\mathrm{Ca}$ residues on the foliage of all plants, leaf samples were not analyzed for Fe. Data for all experiments were analyzed by analysis of variance, with mean separation by the WallerDuncan k-ratio method (SAS, version 9.1; SAS Institute, Cary, NC). 


\section{Results}

In Expt. 1, st. augustinegrass quality was similar for all fertilizer treatments across all evaluation dates, except that unfertilized plots had lower quality ratings in June and May 2002 than plots receiving any fertilizer (Table 2). Turf foliage fertilized with the $8-2-12-4 \mathrm{Mg}$ palm fertilizer had significantly higher concentrations of $\mathrm{N}, \mathrm{P}, \mathrm{K}, \mathrm{Mg}$, and Mn than that receiving the 16-4-8 turf fertilizer (Table 3). St. Augustine grass fertilized with the 16-4-8 turf fertilizer had significantly lower $P$ and $\mathrm{Mg}$ concentrations than unfertilized turf. There were no differences in leaf $\mathrm{Fe}$ or $\mathrm{Zn}$ concentrations among treatments.

In Expt. 2, there were no significant differences in areca palm $\mathrm{N}$ deficiency ratings among fertilized or unfertilized control plants (Table 4 ). Areca palms receiving the 8-2-12$4 \mathrm{Mg}$ palm fertilizer had significantly higher $\mathrm{K}$ deficiency ratings (least amount of $\mathrm{K}$ deficiency) than those receiving higher $\mathrm{N}$ analysis fertilizers (24-2-11 or $12-2-12-4 \mathrm{Mg}$ ) or no fertilizer (Table 4). Potassium deficiency symptoms on areca palms include translucent orange spotting

Table 2. Effects of fertilizer type on visual quality rating of 'Floratam' st. augustinegrass turf growing in a Margate fine sand soil in Davie, FL (Expt. 1).

\begin{tabular}{lcccccc}
\hline & \multicolumn{5}{c}{ Visual quality (1-10 scale) } \\
\cline { 2 - 7 } Fertilizer & $\begin{array}{c}\text { 5 June } \\
\text { 2001 }\end{array}$ & $\begin{array}{c}\text { 6 Aug. } \\
\text { 2001 }\end{array}$ & $\begin{array}{r}\text { 28 Nov. } \\
\text { 2001 }\end{array}$ & $\begin{array}{c}\text { 28 Jan. } \\
\text { 2002 }\end{array}$ & $\begin{array}{r}\text { 20 Mar. } \\
\text { 2002 }\end{array}$ & 3-May-02 \\
\hline None & $6.0 \mathrm{~b}^{\mathrm{y}}$ & $7.6 \mathrm{a}$ & $7.5 \mathrm{a}$ & $7.0 \mathrm{a}$ & $6.9 \mathrm{a}$ & $7.5 \mathrm{~b}$ \\
$16-4-8^{\mathrm{x}}$ & $8.0 \mathrm{a}$ & $8.1 \mathrm{a}$ & $7.6 \mathrm{a}$ & $6.6 \mathrm{a}$ & $7.9 \mathrm{a}$ & $8.4 \mathrm{a}$ \\
$12-2-14-3 \mathrm{Mg}^{\mathrm{w}}$ & $7.9 \mathrm{a}$ & $8.4 \mathrm{a}$ & $7.6 \mathrm{a}$ & $7.3 \mathrm{a}$ & $7.9 \mathrm{a}$ & $8.3 \mathrm{a}$ \\
$8-4-12-4 \mathrm{Mg}^{\mathrm{v}}$ & $7.8 \mathrm{a}$ & $8.0 \mathrm{a}$ & $7.4 \mathrm{a}$ & $7.3 \mathrm{a}$ & $7.5 \mathrm{a}$ & $8.3 \mathrm{a}$ \\
\hline
\end{tabular}

${ }^{\mathrm{z}} \mathrm{l}=$ very poor, 5 = average quality, $10=$ excellent quality.

yean separation within columns by the Waller-Duncan k-ratio method at $P=0.05$

${ }^{x} 16 \mathrm{~N}-1.7 \mathrm{P}-6.7 \mathrm{~K}$

${ }^{\mathrm{w}} 12 \mathrm{~N}-0.9 \mathrm{P}-11.7 \mathrm{~K}-3 \mathrm{Mg}$.

$8 \mathrm{~N}-0.9 \mathrm{P}-10.0 \mathrm{~K}-4 \mathrm{Mg}$.

Table 3. Effects of fertilizer type on nitrogen $(\mathrm{N})$, phosphorus $(\mathbf{P})$, potassium $(\mathrm{K})$, magnesium $(\mathrm{Mg})$, iron $(\mathrm{Fe})$, and manganese $(\mathrm{Mn})$ concentrations of 'Floratam' st. augustinegrass growing in a Margate fine sand soil in Davie, FL after 18 mo. (Expt. 1).

\begin{tabular}{lccccccc}
\hline Fertilizer & $\begin{array}{c}\mathbf{N} \\
\mathbf{( \% )}\end{array}$ & $\begin{array}{c}\mathbf{P} \\
\mathbf{( \% )}\end{array}$ & $\begin{array}{c}\mathrm{K} \\
\mathbf{( \% )}\end{array}$ & $\begin{array}{c}\mathbf{M g} \\
\mathbf{( \% )}\end{array}$ & $\begin{array}{c}\mathrm{Fe} \\
\left(\mathbf{m g} \cdot \mathbf{k g}^{-1}\right)^{\mathbf{y}}\end{array}$ & $\begin{array}{c}\mathbf{M n} \\
\left(\mathbf{m g} \cdot \mathbf{k g}^{-1}\right)\end{array}$ & $\begin{array}{c}\mathbf{Z n}^{-1} \\
\left(\mathbf{m g} \cdot \mathbf{k g}^{-1}\right)\end{array}$ \\
\hline None & $1.70 \mathrm{~b}^{\mathrm{z}}$ & $0.56 \mathrm{a}$ & $1.53 \mathrm{~b}$ & $0.27 \mathrm{a}$ & 73.9 & $7.9 \mathrm{~b}$ & 62.8 \\
$16-4-8^{\mathrm{x}}$ & $1.89 \mathrm{~b}$ & $0.44 \mathrm{~b}$ & $1.82 \mathrm{~b}$ & $0.21 \mathrm{~b}$ & 67.6 & $6.6 \mathrm{~b}$ & 60.9 \\
$8-4-12-4 \mathrm{Mg}^{\mathrm{w}}$ & $2.13 \mathrm{a}$ & $0.55 \mathrm{a}$ & $2.15 \mathrm{a}$ & $0.27 \mathrm{a}$ & 64.5 & $10.7 \mathrm{a}$ & 65.1 \\
\hline
\end{tabular}

${ }^{2}$ Mean separation within columns by the Waller-Duncan k-ratio method at $P=0.05$.

${ }^{\mathrm{y}} 1 \mathrm{mg} \cdot \mathrm{kg}^{-1}=1 \mathrm{ppm}$

${ }^{x} 16 \mathrm{~N}-1.7 \mathrm{P}-6.7 \mathrm{~K}$

${ }^{\mathrm{w}} 8 \mathrm{~N}-0.9 \mathrm{P}-10.0 \mathrm{~K}-4 \mathrm{Mg}$.
Magnesium concentrations in areca palm leaves were lower for those treated with 24-2-11 turf fertilizer than for other fertilizer treatments or unfertilized control plants (Table 5). There were no differences among fertilizer treatments with respect to foliar $\mathrm{Fe}$ concentrations, but palms receiving the $8-2-12-4 \mathrm{Mg}$ palm fertilizer had significantly higher Mn concentrations than unfertilized palms or those receiving the 24-211 turf fertilizer. Palms grown with the 24-2-11 turf fertilizer also had the lowest $\mathrm{Zn}$ and $\mathrm{Cu}$ concentrations, and $8-2-12-4 \mathrm{Mg}$ palm fertilizertreated palms had highest concentrations of these elements.

In Expt. 2 there was a slight but significant reduction in $\mathrm{N}$ deficiency ratings for allamandas receiving no fertilizer, but there were no differences in size or deficiency ratings for $\mathrm{K}$ or $\mathrm{Mg}$ for this species (Table 4). Foliar N concentrations were significantly lower for 8-2-12-4Mg-treated plants than for those receiving other fertilizer treatments or the unfertilized controls (Table 5). There were no differences among treatments in foliar P concentrations. Leaf $\mathrm{K}$ concentrations were highest for 8-2-12$4 \mathrm{Mg}$ and $12-2-12-4 \mathrm{Mg}$ palm fertilizer-treated plants and lowest for unfertilized control plants. Foliar $\mathrm{Mg}$ concentrations were significantly higher for unfertilized control plants than for any fertilized treatments (Table 5).

Allamanda leaf $\mathrm{Fe}$ concentrations were higher for unfertilized control plants than for those receiving the 24-2-11 turf fertilizer or the 8$2-12-4 \mathrm{Mg}$ palm fertilizer (Table 5 ). Manganese concentrations in allamanda leaves were highest for 8-2$12-4 \mathrm{Mg}$-fertilized plants, intermediate for those receiving $12-2-12-4 \mathrm{Mg}$ palm fertilizer, and lowest for unfertilized controls and those receiving the 24-2-11 turf fertilizer. For $\mathrm{Zn}$, the $8-2-12-4 \mathrm{Mg}$ palm fertilizer-treated allamandas had higher leaf concentrations than all other treatments, whereas for $\mathrm{Cu}$, the 24-2-11 turf fertilizer-treated plants had lower leaf concentrations than all other treatments.

There were no significant differences in size or $\mathrm{N}, \mathrm{K}$, or $\mathrm{Mg}$ deficiency ratings for pentas (Table 4 ). Plants receiving the 24-2-11 turf fertilizer had highest foliar $\mathrm{N}$ concentrations, 
followed by those receiving the 82-12-4Mg palm fertilizer, untreated control plants, and those receiving the 12-2-12-4Mg palm fertilizer (Table 5). There were no differences in foliar $\mathrm{P}, \mathrm{K}, \mathrm{Mg}, \mathrm{Fe}, \mathrm{Zn}$, or $\mathrm{Cu}$ concentrations in this species, but pentas receiving the $8-2-12-4 \mathrm{Mg}$ palm fertilizer had significantly higher leaf Mn concentrations than other treatments.

In Expt. 3, cannas exhibited no $\mathrm{N}$ or $\mathrm{Mg}$ deficiency symptoms in any of the treatments, but $\mathrm{K}$ deficiency

Table 4. Effects of fertilizer type on nitrogen $(\mathrm{N})$, potassium $(\mathrm{K})$, and magnesium (Mg) deficiency ratings and plant size of areca palm, pentas, and allamanda grown in a Margate fine sand soil in Davie, FL after 2 years (Expt.2)

\begin{tabular}{|c|c|c|c|c|}
\hline \multirow[b]{2}{*}{ Fertilizer } & \multicolumn{4}{|c|}{ Areca palms } \\
\hline & $\begin{array}{l}\text { N deficiency } \\
(1-5 \text { scale })^{\mathrm{z}}\end{array}$ & $\begin{array}{l}\text { K deficiency } \\
(1-5 \text { scale })^{\mathrm{z}}\end{array}$ & $\begin{array}{l}\text { Mg deficiency } \\
(1-5 \text { scale })^{\mathrm{z}}\end{array}$ & $\begin{array}{l}\text { Total ht } \\
(\mathrm{cm})^{\mathrm{z}}\end{array}$ \\
\hline None & 4.2 & $3.2 \mathrm{~b}^{\mathrm{y}}$ & $4.1 \mathrm{a}$ & 179.6 \\
\hline $24-2-11^{x}$ & 4.4 & $3.2 \mathrm{~b}$ & $3.5 \mathrm{~b}$ & 195.6 \\
\hline $8-2-12-4 \mathrm{Mg}^{\mathrm{w}}$ & 4.4 & $3.9 \mathrm{a}$ & $4.4 \mathrm{a}$ & 195.6 \\
\hline \multirow[t]{3}{*}{$12-2-12-4 \mathrm{Mg}^{\mathrm{v}}$} & 4.3 & $3.4 \mathrm{~b}$ & $3.6 \mathrm{~b}$ & 184.4 \\
\hline & \multicolumn{4}{|c|}{ Pentas } \\
\hline & $\begin{array}{c}\mathrm{N} \text { deficiency } \\
(1-5 \text { scale })\end{array}$ & $\begin{array}{c}\text { K deficiency } \\
(1-5 \text { scale })\end{array}$ & $\begin{array}{c}\text { Mg deficiency } \\
\text { (1-5 scale) }\end{array}$ & $\begin{array}{l}\text { Plant volume } \\
\qquad\left(\mathrm{m}^{3}\right)^{\mathrm{z}}\end{array}$ \\
\hline None & 4.6 & 3.6 & 4.9 & 2.4 \\
\hline $24-2-11$ & 4.6 & 4.1 & 4.8 & 2.8 \\
\hline $8-2-12-4 \mathrm{Mg}$ & 4.7 & 3.9 & 4.9 & 3.0 \\
\hline \multirow[t]{3}{*}{$12-2-12-4 \mathrm{Mg}$} & 4.7 & 3.6 & 4.9 & 2.8 \\
\hline & \multicolumn{4}{|c|}{ Dwarf allamandas } \\
\hline & $\begin{array}{c}\mathrm{N} \text { deficiency } \\
(1-5 \text { scale })\end{array}$ & $\begin{array}{l}\text { K deficiency } \\
(1-5 \text { scale })\end{array}$ & $\begin{array}{c}\text { Mg deficiency } \\
\text { (1-5 scale) }\end{array}$ & $\begin{array}{l}\text { Plant volume } \\
\left(\mathrm{m}^{3}\right)\end{array}$ \\
\hline None & $4.9 \mathrm{~b}$ & 5.0 & 4.8 & 2.6 \\
\hline $24-2-11$ & $5.0 \mathrm{a}$ & 5.0 & 5.0 & 2.5 \\
\hline $8-2-12-4 \mathrm{Mg}$ & $5.0 \mathrm{a}$ & 5.0 & 4.9 & 2.4 \\
\hline $12-2-12-4 \mathrm{Mg}$ & $5.0 \mathrm{a}$ & 5.0 & 5.0 & 2.2 \\
\hline
\end{tabular}

${ }^{2} \mathrm{l}=$ severe deficiency, $3=$ moderate deficiency, $5=$ no deficiency symptoms present $1 \mathrm{~cm}=0.3937 \mathrm{inch}, 1 \mathrm{~m}^{3}=$ $35.3147 \mathrm{ft}^{3}$.

Mean separation within columns and species by the Waller-Duncan k-ratio method at $P<0.05$.

× $24 \mathrm{~N}-0.9 \mathrm{P}-9.2 \mathrm{~K}$

" $8 \mathrm{~N}-0.9 \mathrm{P}-10.0 \mathrm{~K}-4 \mathrm{Mg}$.

${ }^{v} 12 \mathrm{~N}-0.9 \mathrm{P}-10.0 \mathrm{~K}-4 \mathrm{Mg}$.

Table 5. Leaf nitrogen (N), phosphorus (P), potassium (K), magnesium (Mg), iron (Fe), manganese (Mn), zinc (Zn), and copper $(\mathrm{Cu})$ concentrations of areca palm, allamanda, and pentas grown with different fertilizers in a Margate fine sand soil in Davie, FL after 2 years (Expt. 2).

\begin{tabular}{|c|c|c|c|c|c|c|c|c|c|}
\hline Species & Fertilizer & N (\%) & P (\%) & K (\%) & $\operatorname{Mg}(\%)$ & $\begin{array}{c}\mathrm{Fe} \\
\left(\mathrm{mg} \cdot \mathrm{kg}^{-1}\right)^{\mathrm{y}}\end{array}$ & $\begin{array}{c}\mathrm{Mn} \\
\left(\mathrm{mg} \cdot \mathrm{kg}^{-1}\right)\end{array}$ & $\begin{array}{c}\mathrm{Zn} \\
\left(\mathrm{mg} \cdot \mathrm{kg}^{-1}\right)\end{array}$ & $\begin{array}{c}\mathrm{Cu} \\
\left(\mathrm{mg} \cdot \mathrm{kg}^{-1}\right) \\
\end{array}$ \\
\hline \multirow[t]{3}{*}{ Areca palm } & None & $1.86 \mathrm{a}^{\mathrm{z}}$ & $0.94 \mathrm{a}$ & $0.66 \mathrm{~b}$ & $0.19 \mathrm{a}$ & 50.3 & $19.4 \mathrm{~b}$ & $19.5 \mathrm{a}$ & $9.4 \mathrm{ab}$ \\
\hline & $8-2-12-4 \mathrm{Mg}^{\mathrm{w}}$ & $1.66 \mathrm{~b}$ & $0.85 \mathrm{~b}$ & $0.68 \mathrm{~b}$ & $0.19 \mathrm{a}$ & 46.6 & $33.0 \mathrm{a}$ & $22.4 \mathrm{a}$ & $11.3 \mathrm{a}$ \\
\hline & $12-2-12-4 \mathrm{Mg}^{\mathrm{v}}$ & $1.61 \mathrm{~b}$ & $0.92 \mathrm{a}$ & $0.82 \mathrm{a}$ & $0.19 \mathrm{a}$ & 48.3 & $23.8 \mathrm{ab}$ & $13.2 \mathrm{~b}$ & $8.9 \mathrm{ab}$ \\
\hline \multirow[t]{2}{*}{ Allamanda } & None & $3.61 \mathrm{a}$ & 0.20 & $1.60 \mathrm{c}$ & $0.43 \mathrm{a}$ & 80.1 a & $36.9 \mathrm{c}$ & $52.3 \mathrm{~b}$ & 29.9 a \\
\hline & $12-2-12-4 \mathrm{Mg}$ & $3.61 \mathrm{a}$ & 0.20 & $1.90 \mathrm{a}$ & $0.41 \mathrm{~b}$ & $76.2 \mathrm{abc}$ & $63.2 \mathrm{~b}$ & $52.0 \mathrm{~b}$ & $29.4 \mathrm{a}$ \\
\hline \multirow[t]{4}{*}{ Pentas } & None & $2.51 \mathrm{c}$ & 0.24 & 1.74 & 0.53 & 110.6 & $29.0 \mathrm{c}$ & 28.5 & 11.3 \\
\hline & $24-2-11$ & $2.79 \mathrm{a}$ & 0.17 & 1.60 & 0.38 & 113.1 & $33.3 \mathrm{bc}$ & 22.5 & 9.1 \\
\hline & $8-2-12-4 \mathrm{Mg}$ & $2.61 \mathrm{~b}$ & 0.15 & 1.94 & 0.34 & 108.2 & $51.7 \mathrm{a}$ & 28.4 & 10.9 \\
\hline & $12-2-12-4 \mathrm{Mg}$ & $2.40 \mathrm{~d}$ & 0.16 & 1.71 & 0.35 & 110.6 & $37.2 \mathrm{~b}$ & 20.2 & 8.7 \\
\hline
\end{tabular}

${ }^{2}$ Mean separation within columns and species by the Waller-Duncan k-ratio method at $P=0.05$.

${ }^{\mathrm{y}} \mathrm{l} \mathrm{mg} \cdot \mathrm{kg}^{-1}=1 \mathrm{ppm}$.

x $24 \mathrm{~N}-0.9 \mathrm{P}-9.2 \mathrm{~K}$

" $8 \mathrm{~N}-0.9 \mathrm{P}-10.0 \mathrm{~K}-4 \mathrm{Mg}$.

${ }^{v} 12 \mathrm{~N}-0.9 \mathrm{P}-10.0 \mathrm{~K}-4 \mathrm{Mg}$ 
Table 6. Effects of fertilizer type on nitrogen $(\mathrm{N})$, potassium $(\mathrm{K})$, and magnesium $(\mathrm{Mg})$ or iron $(\mathrm{Fe})$ deficiency ratings and plant size of cannas and nandinas grown in Gainesville, FL after 2 years (Expt. 3 ).

\begin{tabular}{|c|c|c|c|c|}
\hline \multirow[b]{2}{*}{ Fertilizer } & \multicolumn{4}{|c|}{ Cannas } \\
\hline & $\begin{array}{l}\mathrm{N} \text { deficiency } \\
(1-5 \text { scale })^{\mathrm{z}}\end{array}$ & $\begin{array}{l}\text { K deficiency } \\
(1-5 \text { scale })^{z}\end{array}$ & $\begin{array}{l}\text { Mg deficiency } \\
(1-5 \text { scale })^{\mathrm{z}}\end{array}$ & $\begin{array}{c}\text { Plant size } \\
\left(\mathrm{m}^{3}\right)\end{array}$ \\
\hline None & 5.00 & $2.89 \mathrm{c}^{\mathrm{y}}$ & 5.00 & $1.13 \mathrm{~b}$ \\
\hline $16-4-8^{x}$ & 5.00 & $3.17 \mathrm{bc}$ & 5.00 & $1.55 \mathrm{~b}$ \\
\hline $8-2-12-4 \mathrm{Mg}^{\mathrm{w}}$ & 5.00 & $3.67 \mathrm{a}$ & 5.00 & $3.43 \mathrm{a}$ \\
\hline \multirow[t]{3}{*}{$12-2-12-4 \mathrm{Mg}^{\mathrm{v}}$} & 5.00 & $3.39 \mathrm{ab}$ & 5.00 & $1.67 \mathrm{~b}$ \\
\hline & \multicolumn{4}{|c|}{ Nandinas } \\
\hline & $\begin{array}{l}\text { N deficiency } \\
(1-5 \text { scale })^{\mathrm{z}}\end{array}$ & $\begin{array}{l}\text { Mg deficiency } \\
(1-5 \text { scale })^{\mathrm{z}}\end{array}$ & $\begin{array}{c}\text { Fe deficiency } \\
(1-5 \text { scale })^{\mathrm{z}}\end{array}$ & $\begin{array}{c}\text { Plant size } \\
\left(\mathrm{m}^{3}\right) \\
\end{array}$ \\
\hline None & 4.83 & 4.72 & $3.22 \mathrm{~b}$ & $0.57 \mathrm{~b}$ \\
\hline $16-4-8$ & 4.89 & 4.94 & $4.33 \mathrm{a}$ & $0.80 \mathrm{a}$ \\
\hline $8-2-12-4 \mathrm{Mg}$ & 4.83 & 4.89 & $4.36 \mathrm{a}$ & $0.99 \mathrm{a}$ \\
\hline $12-2-12-4 \mathrm{Mg}$ & 4.78 & 5.00 & $4.17 \mathrm{a}$ & $0.89 \mathrm{a}$ \\
\hline
\end{tabular}

${ }^{\mathrm{z}} 1=$ severe deficiency, $3=$ moderate deficiency, $5=$ no deficiency symptoms present; $1 \mathrm{~m}^{2}=35.3147 \mathrm{ft}^{2}$.

yean separation within columns and species by the Waller-Duncan k-ratio method at $P<0.05$.

${ }^{x} 16 \mathrm{~N}-1.7 \mathrm{P}-6.7 \mathrm{~K}$.

${ }^{\mathrm{w}} 8 \mathrm{~N}-0.9 \mathrm{P}-10.0 \mathrm{~K}-4 \mathrm{Mg}$.

${ }^{v} 12 \mathrm{~N}-0.9 \mathrm{P}-10.0 \mathrm{~K}-4 \mathrm{Mg}$

Table 7. Leaf nitrogen $(\mathrm{N})$, phosphorus $(\mathrm{P})$, potassium $(\mathrm{K})$, magnesium $(\mathrm{Mg})$, and manganese $(\mathrm{Mn})$ concentrations of canna and nandina grown with different fertilizers in a Bonneau fine sand soil in Gainesville, FL after 2 years (Expt. 3).

\begin{tabular}{llllccc}
\hline Species & Fertilizer & N (\%) & $\mathbf{P ~ ( \% )}$ & $\mathrm{K}(\%)$ & $\mathbf{M g}(\%)$ & $\mathbf{M n}\left(\mathbf{m g} \cdot \mathbf{k g}^{-1}\right)^{\mathbf{y}}$ \\
\hline \multirow{2}{*}{ Nandina } & None & 2.38 & 0.54 & 1.10 & $0.14 \mathrm{a}^{\mathrm{z}}$ & 13.1 \\
& $16-4-8^{\mathrm{x}}$ & 2.12 & 0.51 & 1.25 & $0.12 \mathrm{~b}$ & 14.6 \\
& $8-2-12-4 \mathrm{Mg}^{\mathrm{w}}$ & 2.04 & 0.50 & 1.29 & $0.12 \mathrm{~b}$ & 15.4 \\
& $12-2-12.4 \mathrm{Mg}^{\mathrm{v}}$ & 2.14 & 0.56 & 1.30 & $0.12 \mathrm{~b}$ & 14.4 \\
\hline Canna & None & $2.52 \mathrm{~b}$ & $0.46 \mathrm{ab}$ & 2.49 & $0.55 \mathrm{a}$ & $43.5 \mathrm{~b}$ \\
& $16-4-8$ & $2.26 \mathrm{~b}$ & $0.50 \mathrm{a}$ & 2.56 & $0.41 \mathrm{~b}$ & $67.9 \mathrm{~b}$ \\
& $8-2-12-4 \mathrm{Mg}$ & $2.80 \mathrm{a}$ & $0.51 \mathrm{a}$ & 2.56 & $0.59 \mathrm{a}$ & $152.5 \mathrm{a}$ \\
& $12-2-12-4 \mathrm{Mg}$ & $2.39 \mathrm{~b}$ & $0.42 \mathrm{~b}$ & 2.86 & $0.46 \mathrm{~b}$ & $69.6 \mathrm{~b}$
\end{tabular}

${ }^{2}$ Mean separation within columns and species by the Waller-Duncan k-ratio method at $P=0.05$.

${ }^{\mathrm{y}} 1 \mathrm{mg} \cdot \mathrm{kg}^{-1}=1 \mathrm{ppm}$.

${ }^{x} 16 \mathrm{~N}-1.7 \mathrm{P}-6.7 \mathrm{~K}$.

w $8 \mathrm{~N}-0.9 \mathrm{P}-10.0 \mathrm{~K}-4 \mathrm{Mg}$.

${ }^{v} 12 \mathrm{~N}-0.9 \mathrm{P}-10.0 \mathrm{~K}-4 \mathrm{Mg}$

leaves (Dickey, 1977). Foliar N, P, $\mathrm{K}$, and Mn concentrations were similar among fertilizer treatments for this species, but unfertilized control plants had slightly higher Mg concentrations than unfertilized plants (Table 7).

\section{Discussion}

Fertilizer type generally had no effect on nutrient deficiency symptom severity or size of ornamental dicot plants such as pentas, allamanda, or nandina. In fact, with the exception of Fe deficiency severity and size in nandina, which were probably related, and a very mild $\mathrm{N}$ deficiency in allamanda, unfertilized dicots generally had a quality equivalent to that of the fertilized plants. This suggests that not only are these dicots insensitive to the type of fertilizer they receive, but that under these soil and environmental conditions, they may not benefit greatly from fertilization at all. Gilman and Yeager (1990) and Gilman et al. (2000) concluded that laurel oaks (Quercus hemisphaerica), japanese ligustrum (Ligustrum japonicum), southern magnolia (Magnolia grandiflora), and live oak (Quercus virginiana) growing in similar soils grew as well with or without fertilization. Although foliar Mn concentrations were higher for 8-2-12$4 \mathrm{Mg}$-fertilized allamandas and pentas than for other treatments, these differences were likely because of the more effective sulfate form of $\mathrm{Mn}$ used in the $8-2-12-4 \mathrm{Mg}$ product (Broschat, 1991).
St. augustinegrass also was insensitive to the type of fertilizer used with respect to visual quality, and only during two of the six sampling periods were fertilized plots superior in quality to unfertilized plots. This species is known to be less responsive to fertilization when clippings are not removed (Duble, 1996). The differences between the 16-4-8 and 8-2$12-4 \mathrm{Mg}$ fertilizers in response to $\mathrm{K}$ is likely because of a combination of factors, including the higher percentage of $\mathrm{K}$ in the $8-2-12-4 \mathrm{Mg}$, the lower percentage of $\mathrm{N}$ in the 8-2$12-4 \mathrm{Mg}$, which means that twice as much product and three times as much $\mathrm{K}$ were therefore applied to achieve equivalent $\mathrm{N}$ application rates as the 16-4-8, and all of the $\mathrm{K}$ in the 16-4-8 was water soluble, whereas $100 \%$ of the $\mathrm{K}$ in the $8-2-12-4 \mathrm{Mg}$ was in controlled-release form, making the $\mathrm{K}$ less susceptible to leaching losses during heavy rainfall or irrigation events.

Turf receiving the 16-4-8 turf fertilizer likely had lower foliar $\mathrm{Mg}$ and $\mathrm{Mn}$ concentrations because this product does not contain any $\mathrm{Mg}$ and the Mn sucrate (oxide) is rather ineffective on this soil type (Broschat, 1991). The lower foliar Mg concentrations in the 16-4-8 turf fertilizertreated turf than in unfertilized control turf may be because of a $\mathrm{K}: \mathrm{Mg}$ imbalance or a dilution effect (Mengel and Kirkby, 1979).

Cannas were included in Expt. 3 , which was located in Gainesville, Florida, as a substitute for a palm species, because there are no fastgrowing small palms that are cold hardy at that locality. Cannas are highly susceptible to K deficiency, as are most species of palms (Broschat, $1989,1990,1992)$. In this study, cannas grown with $8-2-12-4 \mathrm{Mg}$ palm fertilizer had less $\mathrm{K}$ deficiency than plants grown with the 16-4-8 turf fertilizer or unfertilized control plants. Those grown with the 12-2$12-4 \mathrm{Mg}$ palm fertilizer were intermediate in this regard. The reasons cited in the previous paragraph on turfgrass likely apply to our results on cannas as well. The size of cannas, however, was greatly affected by fertilizer type, with those grown with 8 $2-12-4 \mathrm{Mg}$ palm fertilizer more than twice the size of all other treatments. Although $\mathrm{K}$ is not known to promote growth in plants, if it is severely 
limiting, it can reduce growth rate or even become fatal in monocots such as palms (Elliott et al., 2004). The reasons why cannas receiving the 82-12-4Mg palm fertilizer had higher $\mathrm{N}$ and $\mathrm{Mg}$ concentrations than those receiving the 16-4-8 turf fertilizer or $12-2-12-4 \mathrm{Mg}$ palm fertilizer are not apparent, given that the 8-2$12-4 \mathrm{Mg}$-treated plants were twice as large as the other treatments. The higher foliar $\mathrm{Mn}$ concentrations in cannas fertilized with the 8-2-12$4 \mathrm{Mg}$ palm fertilizer is likely because of the greater effectiveness of manganese sulfate as a Mn source in this soil type (Broschat, 1991).

Of all the species tested, areca palms were the most sensitive to fertilizer type. During the first year while plants were becoming established, these plants, as well as the pentas in Expt. 2, exhibited primarily $\mathrm{N}$ deficiency symptoms, with no obvious differences among treatments. This was likely caused by the root system being largely confined to the original container root ball. The potting substrate in which these plants had been growing ( 5 pine bark:4 sedge peat: 1 sand by volume) typically requires a high $\mathrm{N}$ fertilization regime to compensate for microbial tie-up of $\mathrm{N}$ in the substrate (Ogden et al., 1987). The maintenance $\mathrm{N}$ application rate used for these plants in this simulated landscape experiment was much lower than that used in the production of these plants in containers. Thus, until the roots become well established in the surrounding landscape soil, which has lower $\mathrm{N}$ requirements, plant growth and quality were likely limited by $\mathrm{N}$ concentrations in the root zone. Additional studies on optimum fertilizer analysis and rates for container-grown plants after transplanting into landscapes or field nurseries are needed.

Once established, our areca palms developed nutrient deficiency symptoms similar to those observed in most landscapes in Florida (Broschat, 2005b). All areca palms showed some $\mathrm{N}$ deficiency, which causes a yelloworange coloration of petioles, rachis, and crownshaft in this species. The reason why unfertilized palms and those receiving the 24-2-11 turf fertilizer had higher foliar $\mathrm{N}$ concentrations than those receiving the 8-2$12-4 \mathrm{Mg}$ or $12-2-12-4 \mathrm{Mg}$ palm fertilizers is not understood because all fertilized palms received the same $\mathrm{N}$ fertilization rate and there were no significant differences in size or $\mathrm{N}$ deficiency severity among treatments.

All palms showed mild to moderate $\mathrm{K}$ deficiency, with palms receiving the $8-2-12-4 \mathrm{Mg}$ palm fertilizer having significantly less $\mathrm{K}$ deficiency than other fertilizer formulations or unfertilized control plants. Furthermore, palms receiving the 24-2-11 turf fertilizer had foliar $\mathrm{K}$ concentrations below those of the unfertilized controls. This may be because of a $\mathrm{N}: \mathrm{K}$ imbalance because a lack of size difference would rule out a dilution effect.

Magnesium deficiency was least severe in unfertilized control palms and in those receiving the $8-2-12$ $4 \mathrm{Mg}$ palm fertilizer. The 24-2-11 turf fertilizer, which contains no $\mathrm{Mg}$, resulted in worse $\mathrm{Mg}$ deficiency than unfertilized control palms. This $\mathrm{Mg}$ deficiency may have been caused by a $\mathrm{K}: \mathrm{Mg}$ or $\mathrm{N}: \mathrm{Mg}$ imbalance (Mengel and Kirkby, 1979). Although palms receiving the $12-2-$ $12-4 \mathrm{Mg}$ were slightly more $\mathrm{Mg}$ deficient than those receiving the 8-2$12-4 \mathrm{Mg}$ palm fertilizer, foliar $\mathrm{Mg}$ concentrations were similar. Palms fertilized with the $12-2-12-4 \mathrm{Mg}$ palm fertilizer received slightly less $\mathrm{Mg}$ than those receiving the 8-2$12-4 \mathrm{Mg}$ palm fertilizer because of its higher $\mathrm{N}$ content.

Foliar micronutrient concentrations were generally lower for palms receiving the 24-2-11 turf fertilizer than for those receiving the 8-2$12-4 \mathrm{Mg}$ palm fertilizer. Although this 24-2-11 turf fertilizer contains $\mathrm{Fe}$ and $\mathrm{Mn}$, they are in sucrate (oxide) forms, which are known to be less effective than the sulfate forms used in the $8-2-12-4 \mathrm{Mg}$ palm fertilizer (Broschat, 1991; Olson, 1951).

These studies suggest that dicot ornamental plants such as nandina, pentas, or allamanda may not benefit from routine maintenance fertilization after establishment, even on infertile sandy soils such as those used in these experiments. Although st. augustinegrass turf appears to have superior quality at certain times of the year when fertilized, the type of fertilizer made no difference visually. However, the use of a high $\mathrm{K}$ product using controlled release $\mathrm{K}$ improved the $\mathrm{K}$ nutritional status of this grass. Tropical monocots such as areca palms and cannas, however, had much higher quality when grown with an $8-2-12-4 \mathrm{Mg}$ palm fertilizer containing $100 \%$ of its $\mathrm{N}, \mathrm{K}$, and $\mathrm{Mg}$ in controlled-release form than with any other fertilizer. Furthermore, palms and cannas receiving high $\mathrm{N}$ turf fertilizers (16-4-8 or 24-2-11) were as deficient in $\mathrm{K}$ as unfertilized control plants, and areca palms receiving turf fertilizer were more deficient in $\mathrm{Mg}$ than unfertilized control palms.

The results of these studies generally support observations by the senior author that palms receiving high $\mathrm{N}: \mathrm{K}$ ratio turf fertilizers, directly or indirectly via fertilization of adjacent turfgrass [roots of large palms can extend out into turfgrass 50 feet or more (T.K. Broschat, personal observations)], have more severe $\mathrm{K}$ or $\mathrm{Mg}$ deficiencies than palms that were never fertilized. Expt. 2 was discontinued after 2 years because the areca palm roots were beginning to approach the far side of the l-ftwide unfertilized buffer strip between plots. Longer-term studies similar to this one with better interplant root isolation should be performed to determine if differences observed between fertilizer treatments become more accentuated with time.

\section{Conclusions}

The use of high $\mathrm{N}: \mathrm{K}$ ratio turf fertilizers appears to have a negative effect on visual quality and nutritional health of areca palms and other ornamental monocots having similar nutritional requirements. A fertilizer containing a lower $\mathrm{N}$ : $\mathrm{K}$ ratio, micronutrients, and controlled-release $\mathrm{N}$, $\mathrm{K}$, and $\mathrm{Mg}$ was more effective in producing healthy palms and cannas than other formulations, and was safe for use on turfgrass and dicot ornamentals, although fertilization of the latter may not be necessary. Thus, if a single fertilizer is to be used in a landscape containing palms, it should not be a high $\mathrm{N}: \mathrm{K}$ ratio, no $\mathrm{Mg}$ product such as those typically applied to turfgrass areas. A lower $\mathrm{N}: \mathrm{K}$ ratio product such as the $8-2-$ $12-4 \mathrm{Mg}$ palm fertilizer could be safely and effectively used on palms, other ornamentals (if warranted), and turfgrass in landscapes that contain palms in Florida sand soils. 


\section{Literature cited}

Broschat, T.K. 1989. Potassium deficiency in south Florida ornamentals. Proc. Florida State Hort. Soc. 102:106109.

Broschat, T.K. 1990. Potassium deficiency of palms in south Florida. Principes 34:151-155.

Broschat, T.K. 1991. Effects of manganese source on manganese uptake by pygmy date palms. HortScience 26: 1389-1391.

Broschat, T.K. 1992. Nutrition of heliconias and related plants. Bul. Heliconia Soc. Int. 6(1-2):20-21.

Broschat, T.K. 2005a. Fertilization of field-grown and landscape palms in Florida. Univ. Florida Environ. Hort. Dept. Circ. ENH1009.

Broschat, T.K. 2005b. Nutrient deficiencies of landscape and field-grown palms in Florida. Univ. Florida Environ. Hort. Dept. Circ. ENH1018.

Broschat, T.K. and M.L. Elliott. 2004. Nutrient distribution and sampling for leaf analysis in St. Augustine grass. Commun. Soil Sci. Plant Anal. 35:23572367.

Dickey, R.D. 1977. Nutritional deficiencies of woody ornamental plants used in Florida landscapes. Univ. Florida Agr. Expt. Sta. Bull. 791.

Duble, R.L. 1996. Turfgrasses. Their management and use in the southern zone, 2nd ed. Texas A\&M University Press, College Station, TX.

Elliott, M.L., T.K. Broschat, J.Y. Uchida, and G.W. Simone. 2004. Compendium of ornamental palm diseases and disorders. APS Press, St. Paul, MN.

Gilman, E.F. 1991. Dispelling misperceptions about trees. Univ. Florida Environ. Hort. Dept. Circ. SSORH3.

Gilman, E.F. and T.H. Yeager. 1990. Fertilizer type and nitrogen rate affects field-grown laurel oak and japanese ligustrum. Proc. Florida State Hort. Soc. 103:370-372.

Gilman, E.F., T.H. Yeager, and D. Kent 2000. Fertilizer rate and type impacts magnolia and oak growth. J. Arboriculture 26:177-182.

Greenberg, A.E.L.S. Clesceri, and A.D. Eaton. 1992. Standard methods for the examination of water and waste water, 17th ed. Amer. Public Health Assn., Washington, DC.

Hach, C.C., B.K. Bowden, A.B. Koplove, and S.V. Brayton. 1987. More powerful peroxide Kjeldahl digestion method. J. Offic. Anal. Chem. 70:783-787.

Knox, G., T. Broschat, and R. Black. 2002. Fertilizer recommendations for landscape plants. Univ. Florida Environ. Hort. Dept. Circ. ENH 858.

Kuo, S. 1996. Phosphorus, p. 869-920. In: J.M. Bartels (ed.). Methods of soil analysis. Part 3. Chemical methods. Soil Sci. Soc. Amer., Madison, WI.
Mengel, K. and E.A. Kirkby. 1979. Principles of plant nutrition, 2 nd ed. Int. Potash Inst., Berne, Switzerland.

Ogden, R.J., F.A. Pokorny, H.A. Mills, and M.G. Dunavent. 1987. Elemental status of pine bark-based potting media. Hort. Rev. 9:103-131.

Olson, R.V. 1951. Effects of acidification, iron oxide addition and other soil treatments on sorghum chlorosis and iron absorption. Soil Sci. Soc. Amer. Proc. 15:97-101.

Soltanpour, P.N., G.W. Johnson, S.M. Workman, J.B. Jones, Jr., and R.O. Miller. 1996. Inductively coupled plasma spectrometry and inductively coupled plasmamass spectroscopy, p. 91-140. In: J.M. Bartels (ed.). Methods of soil analysis. Part 3. Chemical methods. Soil Science Society of America, Madison, WI

Tomlinson, P.B. 1990. The structural biology of palms. Clarendon Press, Oxford, UK

Trenholm, L.E. and J.B. Unruh. 2005a. Warm-season turfgrass response to fertilizer rates and sources. J. Plant Nutr. 28:991-999.

Trenholm, L.E. and J.B. Unruh (eds.). 2005b. Florida lawn handbook, 3rd ed. University Press of Florida, Gainesville, FL.

U.S. Department of Agriculture. 1984. Soil survey of Broward County, Florida. Eastern part. Soil Conservation. Serv., U.S. Dept. of Agriculture, Washington, DC. 